\title{
Long-term outcomes of sutureless and rapid-deployment aortic valve replacement: a systematic review and meta-analysis
}

\author{
Michael L. Williams ${ }^{1,2,3,4}$, Campbell D. Flynn ${ }^{2,5}$, Andrew A. Mamo ${ }^{6}$, David H. Tian ${ }^{2,7}$, Utz Kappert ${ }^{8}$, \\ Manuel Wilbring ${ }^{8}$, Thierry Folliguet ${ }^{9}$, Antonio Fiore', Antonio Miceli ${ }^{10}$, Augusto D'Onofrio ${ }^{11}$, \\ Giorgia Cibin $^{11}$, Gino Gerosa ${ }^{11}$, Mattia Glauber ${ }^{12}$, Theodor Fischlein ${ }^{13}$, Francesco Pollari ${ }^{13}$ \\ ${ }^{1}$ Department of Cardiothoracic Surgery, Royal Prince Alfred Hospital, Sydney, Australia; ${ }^{2}$ The Collaborative Research (CORE) Group, Macquarie \\ University, Sydney, Australia; ${ }^{3}$ The Baird Institute of Applied Heart \& Lung Surgical Research, Sydney, Australia; ${ }^{4}$ The University of Sydney, Sydney, \\ Australia; ${ }^{5}$ Department of Cardiothoracic Surgery, Epworth Hospital, Richmond, Melbourne, Australia; ${ }^{6}$ Department of Cardiology, Prince of Wales \\ Hospital, Sydney, Australia; ${ }^{7}$ Department of Anaesthesia and Perioperative Medicine, Westmead Hospital, Sydney, Australia; ${ }^{8}$ Department of Cardiac \\ Surgery, University Heart Center Dresden, Dresden, Germany; ${ }^{9}$ Department of Cardiac Surgery, Henri Mondor University Hospital, Assistance \\ Publique-Hôpitaux de Paris, Créteil, France; ${ }^{10}$ Minimally Invasive Cardiac Surgery, Istituto Clinico Sant'Ambrogio, Milano, Italy; ${ }^{11}$ Division of \\ Cardiac Surgery, University of Padova, Padova, Italy; ${ }^{12}$ Minimally Invasive Cardiac Surgery Department, Istituto Clinico Sant'Ambrogio - Gruppo \\ San Donato, Milano, Italy; ${ }^{13}$ Cardiac Surgery, Paracelsus Medical University - Klinikum Nürnberg, Nuremberg, Germany \\ Correspondence to: Dr. Michael L. Williams. Department of Cardiothoracic Surgery, Royal Prince Alfred Hospital, Camperdown, NSW, 2050, \\ Australia. Email: dr.michaelwilliams.au@gmail.com.
}

Background: Sutureless and rapid-deployment aortic valve replacement (SURD-AVR) has become a prominent area of research as the medical community evaluate its place amongst other aortic valve interventions. The main advantages of SURD-AVR established to date are the reduced cross-clamp and cardiopulmonary bypass (CPB) times, as well as facilitating minimally invasive surgery in high-risk surgical patients. This current systematic review and meta-analysis, to our knowledge, is the first focusing on longterm outcomes regarding safety, efficacy and durability of SURD-AVR from available current literature.

Methods: A literature search via six electronic databases was performed from their inception to November 2019. Inclusion criteria for this systematic review included survival and postoperative echocardiographic outcomes greater than five years in patients who underwent SURD-AVR with either Perceval or Intuity valves. Studies were identified and data extracted by two independent reviewers. Long-term survival outcomes were aggregated using digitized Kaplan-Meier curves where available.

Results: After applying predefined inclusion and exclusion criteria, four studies were identified for review. All four studies were observational and in total reported data for 1,998 patients. Almost half (42.4\%) of patients underwent SURD-AVR via full sternotomy, with almost one third (30.1\%) also undergoing concomitant cardiac procedures. Aggregate overall survival rates at 1-, 2-, 3-, and 5-year follow-up were 94.9\%, 91.2\%, $89.0 \%$, and $84.2 \%$, respectively. Incidence of strokes (4.8\%), severe paravalvular leaks (PVLs) $(1.5 \%)$ and permanent pacemaker (PPM) insertion (8.2\%) at up to 5-year follow-up were acceptable. At 5-year followup hemodynamic outcomes were also acceptable for mean pressure gradient (MPG) (range, 8.8-13.6 mmHg), peak pressure gradient (PPG) (range, 18.9-21.1 mmHg) and effective orifice area (EOA) (range, $1.5-1.8 \mathrm{~cm}^{2}$ ).

Conclusions: Evaluation of the evidence reporting long-term outcomes of SURD-AVR suggests that it is a safe procedure for AVR with low rates of complications. Long-term outcomes presented in this review show that not only does SURD-AVR have acceptable survival rates, but also good hemodynamic performance at 5 -year follow-up.

Keywords: Sutureless; rapid-deployment; aortic valve replacement (AVR); systematic review

Submitted Feb 24, 2020. Accepted for publication May 05, 2020.

doi: 10.21037/acs-2020-surd-25

View this article at: http://dx.doi.org/10.21037/acs-2020-surd-25 


\section{Introduction}

Aortic stenosis (AS) remains the leading indication for valve replacement surgery in developed countries (1) and the ageing population contributes to increased prevalence of the condition. Surgical aortic valve replacement (AVR) remains a Class I recommendation in low and intermediate risk patients (2). Approximately one third of patients presenting with severe AS are considered too high-risk to proceed with conventional AVR. Morbidity and mortality in isolated conventional AVR have improved over time despite an increase in patient age and comorbid profile (3).

Advances in surgical technology and techniques hope to reduce surgical trauma, thus conferring fewer surgical risks and resulting in shorter hospital stays. Sutureless and rapid deployment (SURD) AVR is beginning to accumulate long-term outcome data demonstrating that they present a safe and effective alternative to conventional AVR surgery (4). Compared to conventional AVR, SURDAVR has similar mortality and complication rates with satisfactory hemodynamic performance. There is benefit in shorter cardiopulmonary bypass (CPB) and cross-clamp durations; both of which are independent risk factors for postoperative morbidity and mortality (5). The advent of transcatheter aortic valve replacement (TAVR) brings technical advances and experience in prosthesis design [and with it, fewer thromboembolic complications and less paravalvular leakage (PVL) compared to earlier prostheses], which has reinvigorated the SURD approach to AVR. Severe AS can be treated by the three aforementioned methods.

Patient selection is a critical factor given the strengths and weaknesses of each approach. A recent meta-analysis (6) compared the three modalities and revealed no differences in 30-day mortality or postoperative stroke between them, but concluded that TAVR and SURD were associated with less postoperative bleeding. SURD was also associated with less acute renal injury, but more conduction disease compared to conventional AVR. However, a limitation of that meta-analysis was that the authors pooled data from studies regardless of differences in surgical risk (i.e., analysis of outcomes in low, intermediate and high-risk patients).

There is a plethora of literature that has evaluated the short-term safety and efficacy of SURD-AVR. However, long-term data of SURD-AVR for outcomes such as survival and hemodynamic performance is lacking. The purpose of this review is to assess the long-term outcomes including safety, clinical efficacy and complications of
SURD-AVR based on available registry data and other case series.

\section{Methods}

\section{Literature search strategy}

Six databases were used to perform electronic searches including Ovid MEDLINE, EMBASE, PubMed, Cochrane Database of Systematic Reviews (CDSR), SCOPUS and Database of Abstracts of Review of Effectiveness (DARE). These databases were searched from their dates of inception to November 2019. The search strategy included a combination of keywords and MeSH headings including "aortic valve replacement OR AVR OR heart valve prosthesis" AND "sutureless OR rapid-deployment" (Supplementary file). Predefined selection criteria were used to assess all relevant articles that were identified.

\section{Selection criteria}

Studies were eligible for inclusion in this systematic review if (I) included patient cohorts which underwent SURDAVR with either the Perceval S (Sorin Group, Saluggia) or Edwards Intuity (Edwards Lifesciences, California) valves, and (II) long-term data (defined as at least 5 years postoperatively) was available for survival rates and echocardiographic performance. Studies were excluded if they did not report complications as endpoints at long-term follow-up. When trials/institutions published duplicate studies with extended lengths of follow-up or a larger study population, only the most updated and complete reports were included for analysis. All studies were limited to those in English and only involving human subjects. Abstracts, case reports, conference presentations, editorials, reviews and expert opinions were excluded.

\section{Data extraction and critical appraisal}

Data was extracted directly from publication texts, tables and figures. Where a trial/investigator had published an extended follow-up article, data regarding earlier reported outcomes were extracted from that group's previous publication when required. All retrieved articles were independently reviewed by two separate investigators (MLW and AAM). Differences of opinion between the two main reviewers were resolved through the means of discussion and consensus, including senior investigators 
if necessary. All retrieved articles had their reference lists reviewed to further identify any other relevant studies. Additional information was sought from corresponding authors when insufficient or indistinct data was identified. The quality of the evidence reported in each included study was assessed using the GRADE system (7).

\section{Outcomes of interest}

The primary outcomes included overall survival and hemodynamic performance at 5-year post SURD-AVR. Secondary outcomes included late and short-term followup incidence rates of postoperative outcomes [including neurological events, permanent pacemaker (PPM) insertion and severe paravalvular leak (PVL)], and operative details including $\mathrm{CPB}$ and cross-clamp times.

\section{Statistical analysis}

Categorical and continuous variables were pooled using meta-analysis of proportions or means, as appropriate, using a random effects model. To facilitate this statistical pooling, means and standard deviations were calculated from the median (with range or interquartile range) where reported using the methods described by Wan and colleagues (8). Pooled data are presented as N (\%) with $95 \%$ confidence intervals ( $95 \% \mathrm{CI}$ ). For outcome data, heterogeneity amongst studies were assessed using the $\mathrm{I}^{2}$ statistic. Thresholds for $\mathrm{I}^{2}$ values were considered as low, moderate and high heterogeneity as $0-49 \%, 50-74 \%$ and $\geq 75 \%$, respectively (9).

The method reported by Guyot and colleagues was used to aggregate overall survival data (10). This method takes digitized Kaplan-Meier survival curves and patient number-at-risk data to reconstruct individual patient data. From this imputed individual patient data, an aggregate survival curve can be created from the pooled cohort data. Echocardiographic results were pooled where $50 \%$ or more of studies reported such results.

All statistical analyses were performed using either Stata (version 16.0, StataCorp, Texas, USA) or R (version 3.5.2, R Foundation for Statistical Computing, Vienna, Austria).

\section{Results}

\section{Quality of studies}

The literature search identified a total of 1,269 studies
(Figure 1). After exclusion of duplicate or irrelevant studies, 47 articles were selected for full-text review. Four studies with a total of 1,998 patients undergoing SURDAVR were deemed suitable for quantitative analysis, with the remaining studies deemed unsuitable predominantly for lacking adequate follow-up periods or duplication of earlier datasets. Two studies did meet inclusion criteria for outcomes, however, these cohorts received the $3 \mathrm{~F}$ Enable (ATS Medical, Minneapolis) SURD valve which is no longer available on the market and therefore were not included.

All four included studies were observational and involved prospectively collected data (11-14) (Table 1). All included studies had patient cohorts of greater than 250 undergoing SURD-AVR (11-14) (Table 1). Note that although 83 patients from the study by Andreas et al., (13) were also included in the TRITON trial (12), the small overlap of patients between these two large cohorts were deemed sufficiently small enough to warrant inclusion of both studies. The Perceval $\mathrm{S}$ valve $(\mathrm{n}=1,211)$ and the Edwards Intuity valve $(\mathrm{n}=787)$ were both used in two studies each (Table 1). Note that the 5 -year results from the Pilot trial (15) were not included as these patients were collated with patients from two other trials in the study by Shrestha and colleagues (11), which are included in this analysis.

The weighted mean follow-up period of all four studies was 2.2 years. All included studies reported mean pressure gradients (MPG) at 5 years postoperatively, whilst two $(11,12)$ and three $(11,12,14)$ studies also reported peak pressure gradient (PPG) and effective orifice area (EOA), respectively. Quality assessment of each study using the GRADE system is seen in Table 1 (7).

\section{Baseline characteristics}

Overall, the weighted pooled age of all patients was 76.2 years [95\% confidence interval (CI), 73.5-78.1]. The patient population was comprised of $43 \%$ males $(95 \% \mathrm{CI}$, 31.9-54.5) with a pooled estimate body mass index (BMI) of $27.8 \mathrm{~kg} / \mathrm{m}^{2}$ (95\% CI, 27.5-28.2). The majority of the patients were in New York Heart Association (NYHA) III or IV class of heart failure (56.4\%; 95\% CI, 39.4-72.7). A small fraction of patients had previously undergone cardiac surgery $(5.5 \%$; 95\% CI, 0.9-13.5). The weighted pooled estimate for the Society of Thoracic Surgery (STS) risk score was 5.6\% (95\% CI, 2.9-7.2). Hypertension, history of 


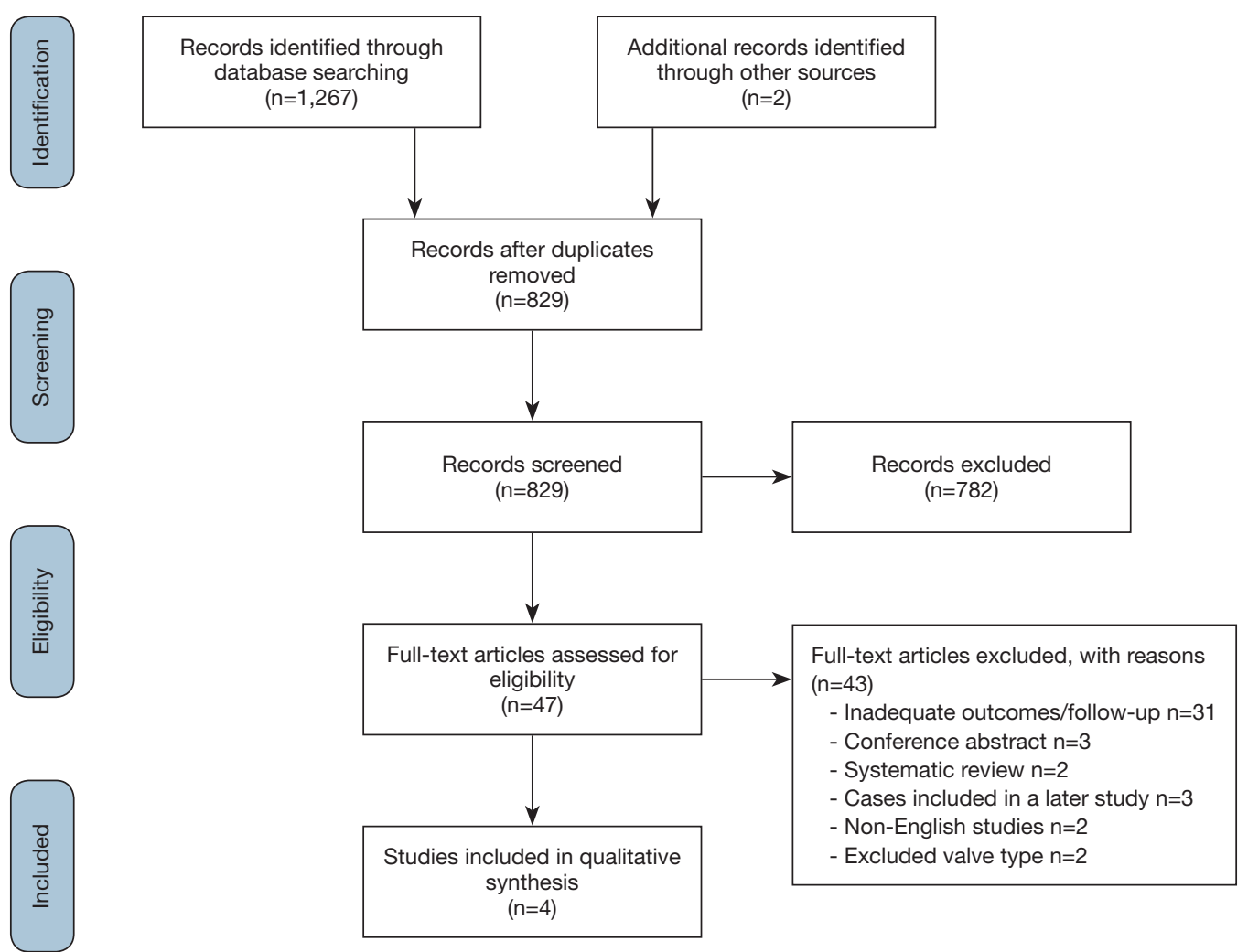

Figure 1 PRISMA flow-chart summarizing the search strategy for relevant publications.

\begin{tabular}{|c|c|c|c|c|c|c|c|c|}
\hline Primary author & Year & Institution(s) & Study period & $\begin{array}{l}\text { Type of } \\
\text { study }\end{array}$ & $\begin{array}{l}\text { Type of } \\
\text { SURD valve }\end{array}$ & $\mathrm{n}$ & $\begin{array}{l}\text { Mean } \\
\text { follow-up time } \\
\text { (years) }\end{array}$ & $\begin{array}{l}\text { GRADE } \\
\text { score }\end{array}$ \\
\hline Shrestha (11) & 2016 & $\begin{array}{l}\text { Pilot, Pivotal and CAVALIER } \\
\text { trial centers ( } 25 \text { centers from } \\
8 \text { European countries) }\end{array}$ & 2007-2012 & OS, P & Perceval S & 731 & $1.8 \pm 0.9^{*}$ & +++ \\
\hline Andreas (13) & 2019 & $\begin{array}{l}\text { Medical University of Vienna, } \\
\text { Vienna, Austria }\end{array}$ & 2010-2017 & OS, P & Intuity & 500 & $1.6 \pm 1.8$ & +++ \\
\hline
\end{tabular}


strokes and peripheral vascular disease were poorly reported in only one study. Baseline characteristics for included studies are summarized in Table 2.

Almost half of the SUDR-AVR were performed through a conventional full sternotomy. Weighted pooled estimates were $42.4 \%$ for full sternotomy, $31.6 \%$ for ministernotomy and $24.9 \%$ of patients underwent right anterior thoracotomy SURD-AVR. Pooled cross-clamp and CPB time were 55 minutes (95\% CI, 42.3-68.6) and 85 minutes (95\% CI, 64.4-108.4), respectively. The proportion of patients undergoing a concomitant procedure along with the SURD-AVR represented almost one third of the patient cohort $(30.1 \%)$, the majority of which was coronary artery bypass grafting (CABG) (17.6\%). Operative details are summarized in Table 3.

\section{Assessment of hemodynamic outcomes}

Pooled proportions of echocardiographic outcomes were unable to be calculated accurately due to inconsistent reporting of follow-up population numbers at the different echocardiographic assessment time-points. All four studies reported MPG at discharge, 1-, 3- and 5-year followup. The pre-operative MPG, PPG and EOA ranged from $42.9 \pm 16.4$ to $49.3 \pm 14.6 \mathrm{mmHg}, 74.0 \pm 25.6$ (preoperative PPG only reported in one study) and $0.70 \pm 0.20$ to $0.75 \pm 0.23 \mathrm{~cm}^{2}$, respectively. Mean reported figures at discharge for MPG ranged from $10.3 \pm 4.4$ to $13.9 \pm$ $4.7 \mathrm{mmHg}$, PPG $20.0 \pm 7.6$ to $20.4 \pm 8.5 \mathrm{mmHg}$ and EOA $1.49 \pm 0.39$ to $1.9 \pm 0.5 \mathrm{~cm}^{2}$. Both $1-$ and 3 -year followup had MPG figures ranging from $8.9 \pm 4.7$ to $11.6 \pm 5.1$ and $7.7 \pm 2.8$ to $12.0 \pm 5.0 \mathrm{mmHg}$, respectively. Ranges for PPG and EOA for the same follow-up time-points were $16.9 \pm 6.1$ to $17.7 \pm 8.0 \mathrm{mmHg}, 16.0 \pm 5.2$ to $17.6 \pm 7.4 \mathrm{mmHg}$, $1.55 \pm 0.37$ to $1.7 \pm 0.5 \mathrm{~cm}^{2}$ and $1.4 \pm 0.4$ to $1.7 \pm 0.2 \mathrm{~cm}^{2}$, respectively. The reported ranges for 5-year follow-up were $8.8 \pm 4.6$ to $13.6 \pm 8.6 \mathrm{mmHg}$ for $\mathrm{MPG}, 18.9 \pm 9.3$ to $21.1 \pm 9.7 \mathrm{mmHg}$ for PPG and $1.5 \pm 0.5$ to $1.80 \pm 0.30 \mathrm{~cm}^{2}$ for EOA. Hemodynamic outcomes for all included studies are summarized in Table 4.

\section{Assessment of safety}

\section{Late follow-up}

Aggregation of overall survival at 1-, 2-, 3-, 4- and 5-year follow-up were $94.9 \%, 91.2 \%, 89.0 \%, 86.9 \%$ and $84.2 \%$, respectively (Figure 2). Weighted pooled estimates for late follow-up (up to 5-year follow-up) overall mortality and

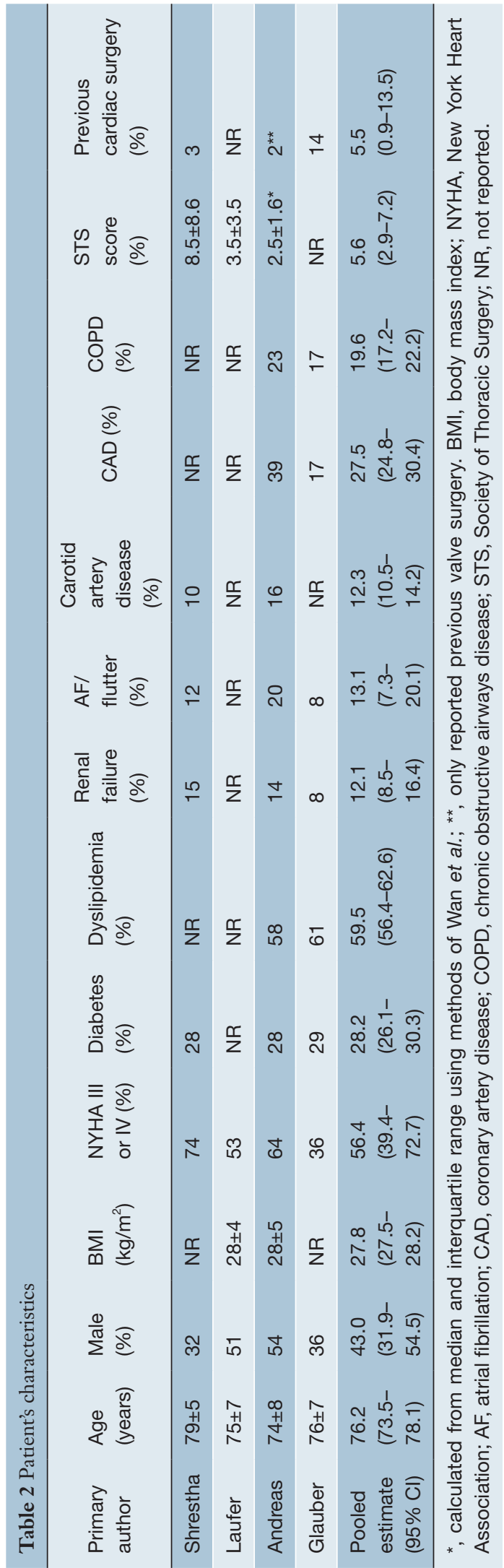



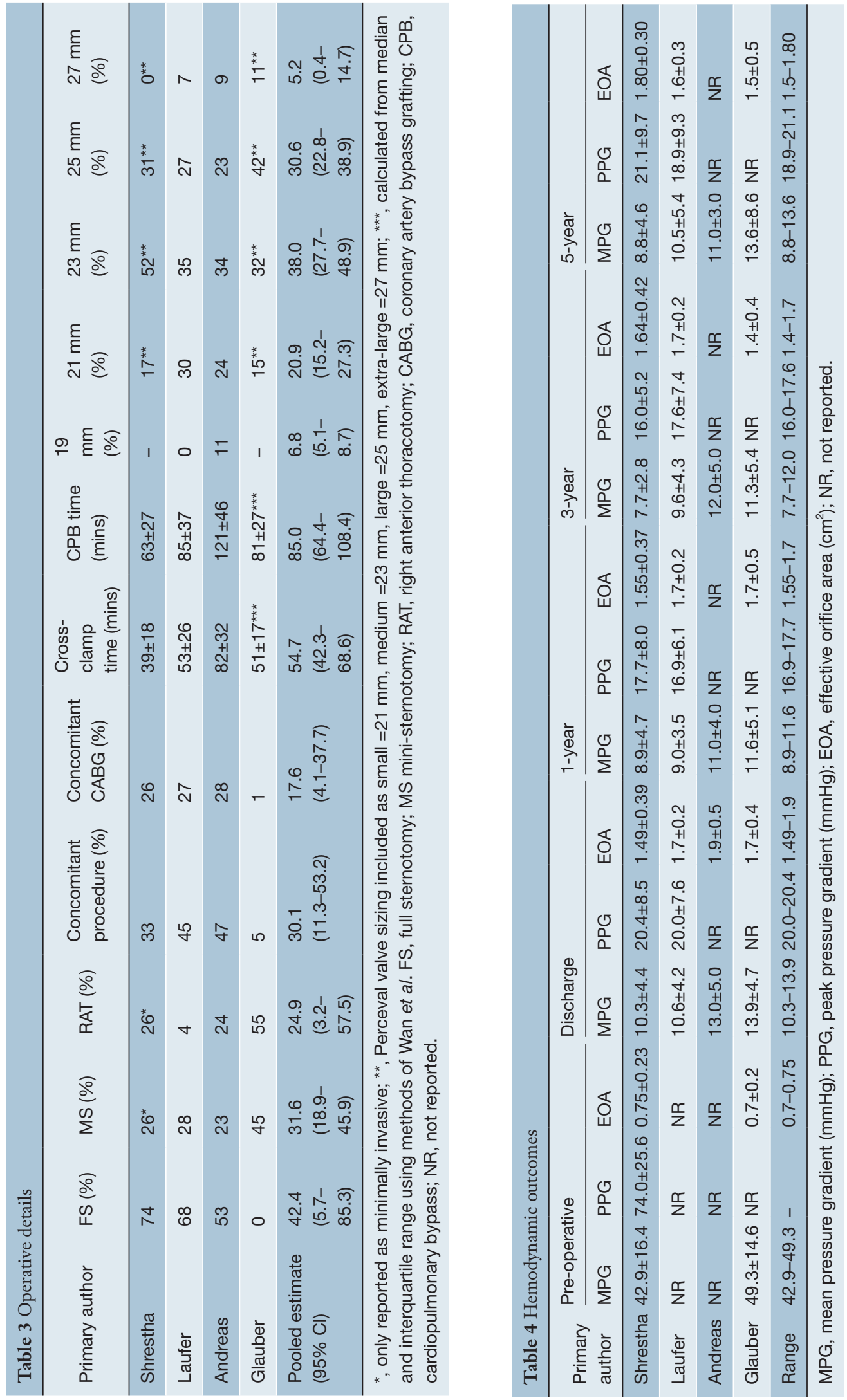


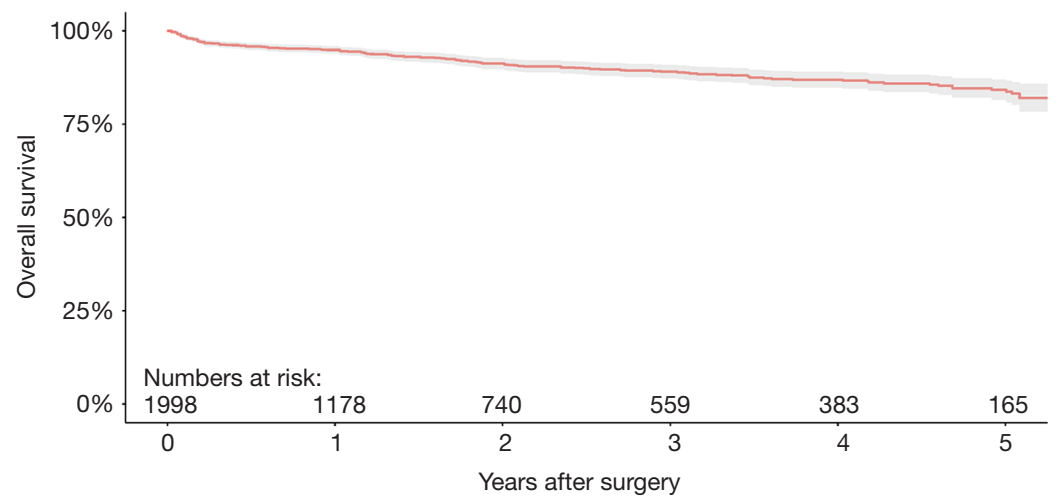

Figure 2 Aggregated overall survival after SURD-AVR. Aggregated from the four included studies via methods described above (shaded region represents $95 \% \mathrm{CI})$.

\begin{tabular}{|c|c|c|c|c|}
\hline Parameter & Events/total & $\mathrm{N}$ & Weighted pooled estimate $(95 \% \mathrm{Cl})$ & Heterogeneity $I^{2}(\%)$ \\
\hline All-cause mortality & $179 / 1,998$ & 4 & $8.9(5.4-13.2)$ & 89 \\
\hline Cardiac-related mortality & $53 / 1,498$ & 3 & $3.7(1.2-7.2)$ & 89 \\
\hline PPM & $160 / 1,998$ & 4 & $8.2(5.9-10.8)$ & 74 \\
\hline Severe PVL & $33 / 1,998$ & 4 & $1.5(0.4-3.0)$ & 81 \\
\hline PVL (all)* & $165 / 1,998$ & 4 & $9.2(2.8-18.7)$ & 97 \\
\hline Valve thrombosis & $0 / 1,518$ & 3 & - & - \\
\hline Endocarditis & $25 / 1,998$ & 4 & $1.1(0.5-1.9)$ & 55 \\
\hline
\end{tabular}

cardiac-related mortality were $8.9 \%$ (95\% CI, 5.4-13.2; $\left.\mathrm{I}^{2}=89 \%\right)$ and $3.7 \%\left(95 \% \mathrm{CI}, 1.2-7.2, \mathrm{I}^{2}=89 \%\right)$, respectively (Table 5). Late neurological events had a pooled estimate of $4.8 \%$ (95\% CI, 2.7-7.6; $\mathrm{I}^{2}=85 \%$ ). There were 25 total cases $\left(1.1 \% ; 95 \% \mathrm{CI}, 0.5-1.9 ; \mathrm{I}^{2}=55 \%\right)$ of endocarditis during late follow-up. Pooled proportions of PPM insertion and valve explantation were $8.2 \%$ (95\% CI, $\left.5.9-10.8 ; \mathrm{I}^{2}=74 \%\right)$ and $2.3 \%\left(95 \% \mathrm{CI}, 1.7-3.1 ; \mathrm{I}^{2}=7 \%\right)$, respectively. Severe PVL was only $1.5 \%$ (95\% CI, 0.4-3.0; $\left.\mathrm{I}^{2}=81 \%\right)$. There were no reported valve thromboses and only eight total cases $\left(0.4 \% ; 95 \% \mathrm{CI}, 0.0-1.1 ; \mathrm{I}^{2}=73 \%\right)$ of structural valve deterioration (SVD) reported during late follow-up.

\section{Early follow-up}

All four studies reported early follow-up ( $<30$ days) mortality rates (Table S1). Weighted pooled estimates of these figures were $1.4 \%$ (95\% CI, 0.4-3.1; $\mathrm{I}^{2}=84 \%$ ) (Table 6). Rates of early neurological events and major bleeding were $2.7 \%\left(95 \% \mathrm{CI}, 1.7-3.8 ; \mathrm{I}^{2}=55 \%\right)$ and $2.4 \%(95 \%$ CI, $\left.0.03-7.6 ; \mathrm{I}^{2}=95 \%\right)$, respectively. There were only two reported cases of endocarditis in one study and rates of valve explantation were $1.1 \%$ (95\% CI, $0.6-1.6 ; \mathrm{I}^{2}=0 \%$ ). Weighted pooled estimate of PPM insertion was $6.0 \%$ 


\begin{tabular}{|c|c|c|c|c|}
\hline All-cause mortality & $36 / 1,998$ & 4 & $1.4(0.4-3.1)$ & 84 \\
\hline Cardiac-related mortality & $20 / 1,498$ & 3 & $1.1(0.2-2.7)$ & 79 \\
\hline PPM & $122 / 1,998$ & 4 & $6.0(4.0-8.4)$ & 77 \\
\hline Thromboembolism & $19 / 1,998$ & 4 & $0.5(0-1.9)$ & 88 \\
\hline Bleeding & $32 / 1,267$ & 3 & $2.4(0.03-7.6)$ & 95 \\
\hline Severe PVL & $13 / 1,498$ & 3 & $0.7(0.1-1.6)$ & 59 \\
\hline Valve thrombosis & $0 / 1,998$ & 4 & - & - \\
\hline SVD & $0 / 1,518$ & 3 & - & - \\
\hline
\end{tabular}

$\mathrm{N}$, number of studies; Cl, confidence interval; PPM, permanent pacemaker; CVA, cerebrovascular accident (stroke and TIA included); PVL, paravalvular leakage; SVD, structural valve deterioration/degeneration.

(95\% CI, 4.0-8.4; $\left.\mathrm{I}^{2}=77 \%\right)$ and severe PVL was $0.7 \%$ (95\% CI, $\left.0.1-1.6 ; \mathrm{I}^{2}=59 \%\right)$, at early follow-up. There were no reported valve thromboses or SVD.

\section{Discussion}

Aortic valve disease, specifically AS, remains the most prevalent valvular pathology within Western countries $(16,17)$. The long-standing treatment for aortic valve disease has been AVR via full median sternotomy (3). However, the treatment of aortic valve disease has become topical again since the introduction of TAVR and minimally invasive surgical approaches in an effort to minimize morbidity and mortality, especially for highrisk surgical patients. Recently, since the introduction of TAVR, the study of SURD-AVR has been reinvigorated since the concept was first explored by Magovern and colleagues in the 1960's (18). Short-term results for SURD-AVR have been published extensively in the literature and show that it is a safe alternative to conventional AVR (19). This is the first review article, to our knowledge, that analyzes long term efficacy, safety and durability of SURD-AVR.

The aggregated 5 -year overall survival of $84.2 \%$ from this current study shows that SURD-AVR is a safe procedure at long-term follow-up. This overall survival compares well with other 5 -year survival rates in the literature, such as the $82.9 \%$ reported by Kvidal and colleagues for conventional AVR in a cohort of over 2,000 patients (20). Pooled proportions of long-term outcomes for all cause and cardiac-related mortality were $8.9 \%$ and $3.7 \%$, respectively, which compare favorably to previous data, such as the $15.0 \%$ all-cause 5-year mortality rate reported by Koene and colleagues (21) for conventional AVR.

Hemodynamic outcomes of SURD valves are a key performance indicator of their efficacy as an alternative to conventional-AVR for the treatment of AS. At present, there is minimal available literature regarding the longterm hemodynamic performance of SURD valves. All included studies in this current review reported MPG, PPG and EOA data at 5 years, which had significantly improved compared to pre-operative figures. Overall, the hemodynamic performance of SURD-AVR long-term appears to be very promising and comparable to other conventional bioprosthetic AVRs $(22,23)$.

SURD-AVR, similarly to conventional AVR, requires excision of the valve and decalcification of the annulus. However, the need for numerous annular sutures is removed and only a small number of guiding sutures are required. Therefore, the main benefits of SURD-AVR are predominantly its advantage of reduced CPB and crossclamp times and facilitating minimally invasive surgical approaches for AVR. There is already substantial evidence in the literature that increased morbidity and mortality in 
cardiac surgery are linked to protracted cross-clamp and CPB durations $(24,25)$. In this meta-analysis, the pooled estimates for cross-clamp and CPB time were 54.7 and 85.0 minutes, respectively. This is slightly higher than those reported in a recent meta-analysis for SURD-AVR which reported cross-clamp and CPB times of 45.6 and 73.1 minutes, respectively (19). However, this meta-analysis by Sian and colleagues only analyzed the Perceval SURD valve, which has been associated with reduced operative times when compared to the Intuity valve in recent studies $(26,27)$. Similar results regarding shorter operative times for the Perceval (range 39-51 minutes cross-clamp and 63-81 minutes $\mathrm{CPB}$ time) compared to the Intuity (53-82 minutes cross-clamp and 85-121 minutes CPB time) valve were also found in this current study. Data regarding isolated and concomitant SURD-AVR cross clamp and CPB times were only available for two of the included studies (data not shown) $(11,13)$. Weighted means of this data were 47.9 and 75.5 minutes for cross-clamp and CPB times, respectively, for isolated SURD-AVR, compared to 69.0 and 103.6 minutes for cross-clamp and CPB times, respectively, for concomitant SURD-AVR. The results seen in this current meta-analysis compare favorably to the crossclamp (78 minutes) and CPB (106 minutes) times from the STS database for conventional isolated AVR with median sternotomy (28).

Complications such as postoperative stroke, valve degeneration, PVL and conduction disorders are other important considerations with any valve-replacement intervention. The incidence of postoperative neurological events were low with pooled rates of $2.7 \%$ at 30 days and $4.8 \%$ at long-term follow-up, which appear to be comparable to previously published incidence of strokes for conventional AVR (3). In comparison, 30-day stroke rates of $3.3 \%$ have been reported for TAVR in a large meta-analysis (29) and recent published evidence of 5-year follow-up TAVR data reports rates of neurological events ranging from $13.2 \%$ to $19.5 \%$ (30,31). Incidence of SVD was low, specifically $0.0 \%$ at 30 days and $0.4 \%$ at late follow-up. PPM insertion has been a focal point of SURD-AVR and has been reported as high as 23\% (32), however the majority of studies within the literature report a much lower range of 6-9\% $(33,34)$. Pooled estimates from this current meta-analysis of $6.0 \%$ at early and $8.2 \%$ at late follow-up for PPM insertion fall within this range reported in the literature. The PPM insertion rates from this current study are also much lower than those reported for TAVR (13.2\%) and acceptable when compared to the
$3.0 \%$ reported for conventional AVR in the meta-analysis by Cao et al. (35). A recent meta-analysis (36) reported PVL incidence of $4.2 \%$ at 30 -day and $5.9 \%$ at 1 -year follow-up after TAVR. In the same study, incidence rates of severe PVL were $0.4 \%$ (30-day) and $0.5 \%$ (1-year follow-up). In the current study, the pooled estimate for severe PVL at 30 days $(0.7 \%)$ is comparable to the rate reported in that study. The pooled estimates of $1.5 \%$ for severe PVL and $9.2 \%$ for all severity PVL at long-term follow-up are higher than those reported by Lazkani et al., however, the follow-up periods are significantly different ( $1 \mathrm{vs}$. up to 5 -year). Some evidence in the literature has suggested that PVL incidence rates are associated with a learning curve for the technique of implantation and there is a significant reduction over time with greater operator experience (5).

There are limitations surrounding this present study which need to be considered when interpreting the above findings. There was significant heterogeneity for a number of outcomes, including mortality, PPM insertion, PVL and neurological events. This could represent the varying degrees of technical experience between institutions and/ or the differences seen between the different types of SURD valves. The included studies also inconsistently reported loss to follow-up. Only one study (14) reported other important outcomes such as total length of stay and length of stay in intensive care. The observational nature of all included studies, and therefore lack of randomization and blinding, present an inherent source of bias in this study. In addition, as mentioned above, two of the included studies $(12,13)$ had a small overlap of patients within their cohorts, creating another possible source of bias. Further studies with long-term results, with minimal loss to follow-up, and randomization comparing SURDAVR to conventional AVR and TAVR are required to further assess the safety and efficacy of SURD-AVR to the aforementioned alternatives.

\section{Conclusions}

In summary, sutureless and rapid-deployment AVR (SURD-AVR) provides a safe and reliable option alongside established therapies in the armamentarium for treatment of AS. Long-term overall survival post SURD-AVR has been proven to be acceptable. Safety and durability of SURD-AVR are also acceptable with low complication rates and good long-term hemodynamic outcomes. Further evidence, such as longer-term studies with more robust 
patient population follow-up, as well as randomized control trials are needed to further assess the clinical outcomes of SURD-AVR.

\section{Expert opinion: rapid deployment valves-time doesn't matter}

\section{Utz Kappert, Manuel Wilbring}

Williams and colleagues report favorable long-term outcomes of rapid deployment aortic valves (RDV) in a systematic review based on nearly 2,000 patients. It is exceedingly helpful to have the positive clinical and hemodynamic results of RDV's confirmed, as the topic of treating high-grade aortic stenosis is a matter of lively debate that has increased in complexity. Instead of one access route and two kinds of prostheses, as it was fifteen years ago, today we have various surgical access routes with many different valve types, complemented by interventional approaches with likewise a large variety of valve prostheses. RDV's on one hand strongly promote minimally invasive techniques for conventional aortic valve replacement, and on the other hand partially diminish the problem of prosthesis-patient-mismatch or indication for rootenlargement procedures. Thus, RDV's have advanced to an essential part of our valve armamentarium. To enhance safety and predictability it is essential to gain best knowledge about the anatomical conditions prior to the procedure. The almost obligatory computed tomography analysis helps to plan access routes, measure annular diameters, choose best prostheses and in general make the operation projectable.

The study by Williams et al. emphasizes the value of RDV's: $84.2 \%$ five-year survival, low rates of severe paravalvular leakage (1.5\%), acceptable pacemaker rates $(8.2 \%)$ and lastly a strong promotion of minimally invasive approaches with a preserved high safety profile. The often-cited time advantage is subordinate-time does not matter.

Points of discussion always include pressure gradients. Williams et al. report satisfactory and stable gradients after five years (Pmean 8.8-13.6 $\mathrm{mmHg}$ ). Of course, there exist prostheses with lower gradients-but the key point is interpretation of gradients. Gradients are multifactorial and depend on prosthesis design and size, hemodynamic situation, afterload, blood pressure or potential hemodilution. If real and comparable gradients are to be determined, a core-lab transthoracic echocardiogram with defined parameters, at adjusted bias parameters and at a defined point in time after the intervention is needed. Unless that is achieved, experience, sizing and resulting clinical performance matters. The excellent durability and clinical performance reported places the whole discussion about gradients into question.

\section{Expert opinion: sutureless and rapid-deployment valves for which patients}

\section{Thierry Folliguet, Antonio Fiore}

SURD valves stem from the intention to offer an alternative to traditional flexible prostheses (stented and stentless biological valves) using conventional open-heart surgery. Several propensity-matched studies and meta-analyses, which compared rapid-deployment sutured and sutureless valves with conventional bioprostheses for AVR, have shown superiority of these valves in terms of surgical times, perioperative atrial fibrillation, transfusion rates, and intensive care unit and hospital stay except for post pacemaker implantation (37).

Studies confirm that these valves may offer additional benefits for patients with a small aortic annulus or in patients with aortic wall abnormalities, such as a calcified aortic root (38).

A recent prospective and randomized study, the PERSIST-AVR trial, demonstrates the superiority of the sutureless valve over stented valves for procedural times, either in isolated or combined procedures (39). Combined procedures such as double valves or AVR and coronary artery bypass grafting can be challenging in elderly patients with longer $\mathrm{CPB}$ times resulting in increased morbidity such as acute renal failure. Reducing the $\mathrm{CPB}$ and cross clamp time is a major advantage of the SURD valves for these patients.

Minimally invasive techniques are greatly facilitated by the design of these valves in addition to low rates of reoperation, which make these valves an important addition to surgical AVR technologies $(5,14)$.

\section{Expert opinion: how long is a long time?}

\section{Antonio Miceli}

A number of studies have demonstrated the advantages of SURD valves in terms of 30-day mortality and morbidities, especially in the minimally invasive setting $(28,40,41)$. Nevertheless, few papers have focused on long-term 
outcomes. In this issue of the Annals of Cardiothoracic Surgery, Williams et al. used meta-analysis to investigate the safety, efficacy and durability of SURD-AVR from current literature. As expected, out of 1,267 papers screened, only four were identified for analysis, highlighting the lack of data. Results suggest the use of SURD valves over conventional sutured valves as they reduce operative times, facilitate minimally invasive approaches and long-term outcomes do not differ from other valves. Specifically, overall survival at 1, 3, and 5 years were $94.9 \%, 89 \%$ and $84 \%$ respectively, with acceptable rate of stoke $(4.8 \%)$, paravalvular leakage $(1.5 \%)$ and permanent pacemaker implantation $(8.2 \%)$ up to five years. However, the limited sample size limits the accuracy of these data. The percentage of patients at risk for survival was 59\%, 28\% and $8 \%$ at $1-, 3$ - and 5 -year, respectively, revealing that only 165 patients were available for survival analysis at 5 -year. Furthermore, long-term secondary outcomes were related to events occurred $>30$ days and expressed as linearized event rates per patient year (\% ppy) of follow-up. Therefore, late postoperative outcomes reported with \% (events/total) in Table 5, do not reflect the real percentage of complications because of the low number of patients at risk (total is not 1,998). Finally, the weighted mean follow-up of these studies was only 2.2 years, which is far from the conventional 10 -year outcomes. Structural valve degeneration and hemodynamic performances are the main key factors for judging the valve durability. Perceval and Intuity valves represent an evolution of well-recognized valves such as Pericarbon and Perimount Magna Ease, which have provided excellent long-term durability $(42,43)$. The associated sutureless/rapid deployment technology has made AVR a safe and fast implantation procedure with no trauma on the aortic annulus and leaflets. The combination of these factors should guarantee the long-term durability. However, studies with accurate longer follow-up are required. The results of the Perceval Sutureless Implant vs Standard (PERSIST) AVR controlled randomized trial will give us detailed information on 5-year outcomes defining the future of these valves.

\section{Expert opinion: aortic valve bioprostheses: a wide range of choices requiring a selection algorithm}

\section{Augusto D’Onofrio, Giorgia Cibin, Gino Gerosa}

The introduction of SURD-AVR has expanded the portfolio of aortic valve substitutes giving surgeons another option for patients with aortic valve stenosis requiring a biological prosthesis. Conventional stented prostheses and SURD-AVR enable surgeons to implant an aortic valve prosthesis with conventional surgery (CS) and minimally invasive cardiac surgery (MICS) while transcatheter (TAVR) devices are implanted through a "micro-invasive approach" ( $\mu$-ICS), meaning no cardiopulmonary bypass, on the beating heart, completely percutaneously (or with a very small skin incision) and possibly with no general anesthesia (44). With so many options the choice of the most appropriate device becomes crucial in order to tailor every procedure to the single patient and to optimize results. This selection algorithm should take into consideration several aspects like surgical risk profile, clinical and anatomical characteristics and patient's preferences. The first step of this process is to select among CS, MICS or $\mu$-ICS. Once CS or MICS have been selected then the choice is between conventional bioprostheses or SURD-AVR. As far as SURD-AVR is concerned, our task is to identify which patients will benefit more from SURDAVR and, which patients should not receive SURD-AVR. This paper by Dr. Williams and colleagues, highlights the main advantages and disadvantages of SURD and gives us more insights into the knowledge of these relatively new devices. Based on Dr. William's findings and also on our experience, we believe that SURD-AVR is indicated in patients requiring short surgical times, in those scheduled for minimally invasive and/or combined procedures and in those with small aortic annulus (due to remarkably low gradients, especially in small sizes). On the other hand, SURD-AVR should not be performed in patients with pre-existing conduction disorders (due to the high risk of postoperative permanent pacemaker implantation), in those with a need for valve oversizing (due to the risk of prosthesis displacement with consequent severe paravalvular leak) and in those with unfavorable anatomy like asymmetric bulky calcifications, bicuspid aortic valve, intramuscular calcifications, very oval and asymmetric annulus (due to the risk of paravalvular leak).

\section{Expert opinion: SURD valve durability: the younger the better}

\section{Mattia Glauber}

Williams et al. have completed a meta-analysis on the available literature investigating the durability of SURD 
valves. Safety, efficacy and hemodynamic performance have been largely demonstrated by several short and medium term studies. The advantages have proven to be more evident in the presence of a minimally invasive cardiothoracic surgery (MICS) approach. However research and results on long-term outcomes remains poor and the lack of data is confirmed by the fact that only four studies qualified for analysis from 1,267 screened papers.

The interesting outcome of this meta-analysis is that long-term results of SURD valves do not differ from traditional sutured valves. However, they are recommended over standard valves as they provide shorter operative time and facilitate MICS procedures. It is well known that valve durability is influenced by hemodynamic performance, freedom from structural valve degeneration (SVD), PVL, thrombosis and endocarditis; moreover cross-clamp and cardiopulmonary bypass time, pacemaker implant and prosthesis mis-match play a role in survival.

Meuris et al. report the 5-year clinical and hemodynamic outcomes. Overall 5 -year survival was $72.3 \%$, and freedom from endocarditis and atrioventricular block was $90.7 \%$ and $91 \%$, respectively. Additionally, no hemolysis, stroke, or valve thrombosis, dislodgment or SVD was observed within the 5-year follow-up (15). Shrestha and colleagues confirmed this, reporting midterm clinical and hemodynamic results on 731 patients undergoing aortic valve replacement in twenty-five European centers. Most important, neither valve migration, SVD or thrombosis was observed at 5-year follow-up. In addition, the rate of major PVL was $1.4 \%$ and $1 \%$ at early and late follow-ups (45).

Following evidence on the safety and durability of SURD bioprostheses out to midterm follow-up, it is reasonable to consider shifting the age limit to younger patients given reoperative surgery has a lower risk if the first procedure is performed through a MICS approach and the potential of a future valve in valve ( $\mathrm{ViV}$ ) solution (46).

SURD valves have proven to be safe and suitable for ViV due to the high radiopacity, low risk of coronary obstruction and the expandable stent of Perceval that allows larger-sized transcatheter aortic valves with lower postimplant gradients $(47,48)$.

Ten-year durability follow-up data of SURD valves is still missing however the actual data looks promising. Treating disease by surgical removal of the native valve still represents the best therapy for a wide number of young, low and medium risk patients affected by degenerative or congenital valve disease. Whether SURD valves may be considered, especially when combined with MICS, the best alternative to conventional sutured prostheses and transcatheter aortic valves has yet to be confirmed by wider studies.

\section{Expert opinion: the lesson of SURD-AVR's outcomes: the more tailored, the better}

\section{Theodor Fischlein, Francesco Pollari}

The metanalysis of Williams and colleagues investigated early and long-term outcomes of SURD prostheses. In addition to the findings regarding long-term survival (up to 5 years in this study), the low rate of surgical complications is also remarkable. It seems that after a learning phase and a better understanding of risk factor and application possibilities, the complication rate following SURD-AVR reduced significantly over the years. The basic lesson is that we should learn to exploit the features of each prosthesis. On one hand, the sutureless prosthesis provides significant advantages in small and severely calcified annuli, as well as in mini-thoracotomy cases and redo-AVR. On the other hand, due to its different structure, the rapid deployment prosthesis can simplify the operation in case of combined procedures with coronary revascularization (when many proximal anastomoses on the ascending aorta are needed) or in case of ascending/root dilatation. The big advantage of surgery is precisely the possibility to adapt and provide each patient with an individual treatment in order to achieve a low complication rate. The most striking example is the reduction of postoperative pacemaker implantation (PPI) after SURD-AVR. The recent introduction of a new version of the sutureless prosthesis, the Perceval Plus (LivaNova PLC., London, United Kingdom), with a thinner annular portion, in association with other surgical refinements (49), did dramatically reduce the incidence of PPI. Indeed, the high heterogeneity in the present metanalysis reflects this phenomenon. This achievement demonstrates how important and worthwhile can be a critical analysis of outcomes, such as the presented work of Williams and colleagues, which brings a precious light on the early and long-term outcomes of SURD-AVR.

\section{Acknowledgments}

None.

\section{Footnote}

Conflicts of Interest: The authors have no conflicts of interest 
to declare.

Open Access Statement: This is an Open Access article distributed in accordance with the Creative Commons Attribution-NonCommercial-NoDerivs 4.0 International License (CC BY-NC-ND 4.0), which permits the noncommercial replication and distribution of the article with the strict proviso that no changes or edits are made and the original work is properly cited (including links to both the formal publication through the relevant DOI and the license). See: https://creativecommons.org/licenses/by-nc$\mathrm{nd} / 4.0 /$.

\section{References}

1. Kang DH, Park SJ, Lee SA, et al. Early Surgery or Conservative Care for Asymptomatic Aortic Stenosis. N Engl J Med 2020;382:111-9.

2. Nishimura RA, Otto CM, Bonow RO, et al. 2017 AHA/ACC Focused Update of the 2014 AHA/ACC Guideline for the Management of Patients With Valvular Heart Disease: A Report of the American College of Cardiology/American Heart Association Task Force on Clinical Practice Guidelines. J Am Coll Cardiol 2017;70:252-89.

3. Brown JM, O'Brien $\mathrm{SM}, \mathrm{Wu} \mathrm{C}$, et al. Isolated aortic valve replacement in North America comprising 108,687 patients in 10 years: Changes in risks, valve types, and outcomes in the Society of Thoracic Surgeons National Database. J Thorac Cardiovasc Surg 2009;137:82-90.

4. Di Eusanio M, Phan K, Berretta P, et al. Sutureless and Rapid-Deployment Aortic Valve Replacement International Registry (SURD-IR): early results from 3343 patients†. Eur J Cardiothorac Surg 2018;54:768-73.

5. Phan K, Tsai YC, Niranjan N, et al. Sutureless aortic valve replacement: a systematic review and meta-analysis. Ann Cardiothorac Surg 2015;4:100-11.

6. Lloyd D, Luc JGY, Indja BE, et al. Transcatheter, sutureless and conventional aortic-valve replacement: a network meta-analysis of 16,432 patients. J Thorac Dis 2019;11:188-99.

7. Balshem H, Helfand M, Schunemann HJ, et al. GRADE guidelines: 3. Rating the quality of evidence. J Clin Epidemiol 2011;64:401-6.

8. Wan X, Wang W, Liu J, et al. Estimating the sample mean and standard deviation from the sample size, median, range and/or interquartile range. BMC Med Res Methodol 2014;14:135.
9. Higgins JP, Thompson SG, Deeks JJ, et al. Measuring inconsistency in meta-analyses. BMJ 2003;327:557-60.

10. Guyot P, Ades AE, Ouwens MJ, et al. Enhanced secondary analysis of survival data: reconstructing the data from published Kaplan-Meier survival curves. BMC Med Res Methodol 2012;12:9.

11. Shrestha M, Fischlein T, Meuris B, et al. European multicentre experience with the sutureless Perceval valve: clinical and haemodynamic outcomes up to 5 years in over 700 patients. Eur J Cardiothorac Surg 2016;49:234-41.

12. Laufer G, Haverich A, Andreas M, et al. Long-term outcomes of a rapid deployment aortic valve: data up to 5 years. Eur J Cardiothorac Surg 2017;52:281-7.

13. Andreas M, Coti I, Rosenhek R, et al. Intermediate-term outcome of 500 consecutive rapid-deployment surgical aortic valve proceduresdagger. Eur J Cardiothorac Surg 2019;55:527-33.

14. Glauber M, Di Bacco L, Cuenca J, et al. Minimally Invasive Aortic Valve Replacement with Sutureless Valves: Results From an International Prospective Registry. Innovations (Phila) 2020;15:120-30.

15. Meuris B, Flameng WJ, Laborde F, et al. Five-year results of the pilot trial of a sutureless valve. J Thorac Cardiovasc Surg 2015;150:84-8.

16. Maganti K, Rigolin VH, Sarano ME, et al. Valvular heart disease: diagnosis and management. Mayo Clin Proc 2010;85:483-500.

17. Nkomo VT, Gardin JM, Skelton TN, et al. Burden of valvular heart diseases: a population-based study. Lancet 2006;368:1005-11.

18. Magovern GJ, Cromie HW. Sutureless Prosthetic Heart Valves. J Thorac Cardiovasc Surg 1963;46:726-36.

19. Sian K, Li S, Selvakumar D, et al. Early results of the Sorin $((\mathrm{R}))$ Perceval S sutureless valve: systematic review and meta-analysis. J Thorac Dis 2017;9:711-24.

20. Kvidal P, Bergstrom R, Horte LG, et al. Observed and relative survival after aortic valve replacement. J Am Coll Cardiol 2000;35:747-56.

21. Koene BM, Soliman Hamad MA, Bouma W, et al. Impact of prosthesis-patient mismatch on early and late mortality after aortic valve replacement. J Cardiothorac Surg 2013;8:96

22. van der Straaten EP, Rademakers LM, van Straten $\mathrm{AH}$, et al. Mid-term haemodynamic and clinical results after aortic valve replacement using the Freedom Solo stentless bioprosthesis versus the Carpentier Edwards Perimount stented bioprosthesis. Eur J Cardiothorac Surg 2016;49:1174-80. 
23. Dalmau MJ, Gonzalez-Santos JM, Blazquez JA, et al. Hemodynamic performance of the Medtronic Mosaic and Perimount Magna aortic bioprostheses: five-year results of a prospectively randomized study. Eur J Cardiothorac Surg 2011;39:844-52; discussion 852.

24. Iino K, Miyata H, Motomura N, et al. Prolonged CrossClamping During Aortic Valve Replacement Is an Independent Predictor of Postoperative Morbidity and Mortality: Analysis of the Japan Cardiovascular Surgery Database. Ann Thorac Surg 2017;103:602-9.

25. Salis S, Mazzanti VV, Merli G, et al. Cardiopulmonary bypass duration is an independent predictor of morbidity and mortality after cardiac surgery. J Cardiothorac Vasc Anesth 2008;22:814-22.

26. Berretta P, Andreas M, Carrel TP, et al. Minimally invasive aortic valve replacement with sutureless and rapid deployment valves: a report from an international registry (Sutureless and Rapid Deployment International Registry) dagger. Eur J Cardiothorac Surg 2019;56:793-9.

27. D'Onofrio A, Salizzoni S, Filippini C, et al. Surgical aortic valve replacement with new-generation bioprostheses: Sutureless versus rapid-deployment. J Thorac Cardiovasc Surg 2019. [Epub ahead of print].

28. Miceli A, Santarpino G, Pfeiffer S, et al. Minimally invasive aortic valve replacement with Perceval S sutureless valve: early outcomes and one-year survival from two European centers. J Thorac Cardiovasc Surg 2014;148:2838-43.

29. Eggebrecht H, Schmermund A, Voigtlander T, et al. Risk of stroke after transcatheter aortic valve implantation (TAVI): a meta-analysis of 10,037 published patients. EuroIntervention 2012;8:129-38.

30. Barbanti M, Tamburino C, D'Errigo P, et al. Five-Year Outcomes of Transfemoral Transcatheter Aortic Valve Replacement or Surgical Aortic Valve Replacement in a Real World Population. Circ Cardiovasc Interv 2019;12:e007825.

31. Makkar RR, Thourani VH, Mack MJ, et al. Five-Year Outcomes of Transcatheter or Surgical Aortic-Valve Replacement. N Engl J Med 2020;382:799-809.

32. Bouhout I, Mazine A, Rivard L, et al. Conduction Disorders After Sutureless Aortic Valve Replacement. Ann Thorac Surg 2017;103:1254-60.

33. Folliguet TA, Laborde F, Zannis K, et al. Sutureless perceval aortic valve replacement: results of two European centers. Ann Thorac Surg 2012;93:1483-8.

34. Dalén M, Biancari F, Rubino AS, et al. Ministernotomy versus full sternotomy aortic valve replacement with a sutureless bioprosthesis: a multicenter study. Ann Thorac Surg 2015;99:524-30.

35. Cao C, Ang SC, Indraratna P, et al. Systematic review and meta-analysis of transcatheter aortic valve implantation versus surgical aortic valve replacement for severe aortic stenosis. Ann Cardiothorac Surg 2013;2:10-23.

36. Lazkani M, Yerasi C, Prakash S, et al. Permanent pacemaker implantation and paravalvular leak rates following sutureless aortic valve operations. J Card Surg 2018;33:808-17.

37. Berretta P, Arzt S, Fiore A, et al. Current trends of sutureless and rapid deployment valves: an 11-year experience from the Sutureless and Rapid Deployment International Registry. Eur J Cardiothorac Surg 2020. [Epub ahead of print].

38. Gersak B, Fischlein T, Folliguet TA, et al. Sutureless, rapid deployment valves and stented bioprosthesis in aortic valve replacement: recommendations of an International Expert Consensus Panel. Eur J Cardiothorac Surg 2016;49:709-18.

39. Lorusso R, Folliguet T, Shrestha M, et al. Sutureless versus Stented Bioprostheses for Aortic Valve Replacement: The Randomized PERSIST-AVR Study Design. Thorac Cardiovasc Surg 2020;68:114-23.

40. Miceli A, Antonio L, Glauber M. Sutureless valves: A future without sutures. J Thorac Cardiovasc Surg 2016;151:1627-8.

41. Glauber M, Ferrarini M, Miceli A. Minimally invasive aortic valve surgery: state of the art and future directions. Ann Cardiothorac Surg 2015;4:26-32.

42. Folliguet TA, Le Bret E, Bachet J, Laborde F. Pericarbon pericardial bioprosthesis: an experience based on the lessons of the past. Ann Thorac Surg 2001;71:S289-S292.

43. McClure RS, Narayanasamy N, Wiegerinck E, et al. Late outcomes for aortic valve replacement with the Carpentier-Edwards pericardial bioprosthesis: up to 17-year follow-up in 1,000 patients. Ann Thorac Surg 2010;89:1410-6.

44. D'Onofrio A, Gerosa G. Technique versus technology and the (r)evolution of cardiac surgery: a professional journey from classical surgery to embracing intervention. Eur J Cardiothorac Surg 2017;52:835-7.

45. Shrestha M, Fischlein T, Meuris B, et al. European multicentre experience with the sutureless Perceval valve: clinical and haemodynamic outcomes up to 5 years in over 700 patients. Eur J Cardiothorac Surg 2016;49:234-41.

46. Santarpino G, Berretta P, Kappert U, et al. Minimally 
Invasive Redo Aortic Valve Replacement: Results From a Multicentric Registry (SURD-IR). Ann Thorac Surg 2020. [Epub ahead of print].

47. Mangner N, Holzhey D, Misfeld M, Linke A. Treatment of a degenerated sutureless Sorin Perceval ${ }^{\circledR}$ valve using an Edwards SAPIEN 3. Interact Cardiovasc Thorac Surg 2018;26:364-6.

Cite this article as: Williams ML, Flynn CD, Mamo AA, Tian DH, Kappert U, Wilbring M, Folliguet T, Fiore A, Miceli A, D'Onofrio A, Cibin G, Gerosa G, Glauber M, Fischlein T, Pollari F. Long-term outcomes of sutureless and rapiddeployment aortic valve replacement: a systematic review and meta-analysis. Ann Cardiothorac Surg 2020;9(4):265-279. doi: 10.21037/acs-2020-surd-25
48. Landes U, Dvir D, Schoels W, et al. TCT- 160 Valve-in-Valve Transcatheter Aortic Implantation in Degenerative Sutureless Bioprostheses. J Am Coll Cardiol 2017;70:B69.

49. Vogt F, Moscarelli M, Nicoletti A, et al. Sutureless Aortic Valve and Pacemaker Rate: From Surgical Tricks to Clinical Outcomes. Ann Thorac Surg 2019;108:99-105. 


\section{Example search string used for database searches}

(I) Aortic Valve/or Aortic Valves Stenosis/

(II) Heart Valve Prosthesis/or Heart Valve Prosthesis Implantation/

(III) Aortic valve replacement* or AVR*

(IV) Sutureless*

(V) Rapid-deployment* or rapid deployment*

(VI) SURD*

(VII) 1 or 2 or 3

(VIII) 4 or 5 or 6

(IX) 7 and 8

(X) Remove duplicates from 9

\begin{tabular}{|c|c|c|c|c|c|c|c|c|c|c|c|c|c|c|}
\hline $\begin{array}{l}\text { Primary } \\
\text { author }\end{array}$ & $\begin{array}{c}\text { Mortality } \\
(\%)\end{array}$ & $\begin{array}{c}\text { Cardiac } \\
\text { death (\%) }\end{array}$ & $\begin{array}{c}\text { PPM } \\
(\%)\end{array}$ & $\begin{array}{l}\mathrm{AF} \\
(\%)\end{array}$ & $\begin{array}{l}\text { CVA } \\
(\%)\end{array}$ & $\begin{array}{l}\text { TBB } \\
(\%)\end{array}$ & $\begin{array}{c}\text { Bleeding } \\
(\%)\end{array}$ & $\begin{array}{l}\text { TP } \\
(\%)\end{array}$ & $\begin{array}{c}\text { Explants } \\
(\%)\end{array}$ & $\begin{array}{c}\text { Endo- } \\
\text { carditis } \\
(\%)\end{array}$ & $\begin{array}{c}\text { Hemolysis } \\
\text { (\%) }\end{array}$ & $\begin{array}{c}\text { Valve } \\
\text { thrombosis } \\
(\%)\end{array}$ & SVD & $\begin{array}{c}\text { Severe } \\
\text { PVL (\%) }\end{array}$ \\
\hline Shrestha & 3.4 & 1.9 & 6.0 & NR & 1.6 & 2.3 & NR & NR & 1.4 & 0.3 & 0.5 & 0 & 0 & 1.4 \\
\hline Laufer & 1.7 & 1.7 & 6.6 & NR & 3.8 & 0.3 & 7.7 & NR & 1.4 & 0 & 0 & 0 & 0 & 0.7 \\
\hline Andreas & 0.8 & NR & 8.6 & NR & 3.6 & 0 & 0.2 & NR & 1.0 & 0 & NR & 0 & 0 & NR \\
\hline Glauber & 0.4 & 0.2 & 3.3 & NR & 2.3 & 0.2 & 1.9 & 0.4 & 0.6 & 0 & NR & 0 & NR & 0.2 \\
\hline $\begin{array}{l}\text { Weighted } \\
\text { estimate } \\
(95 \% \mathrm{Cl})\end{array}$ & $\begin{array}{c}1.4 \\
(0.4-3.1)\end{array}$ & $\begin{array}{c}1.1 \\
(0.2-2.7)\end{array}$ & $\begin{array}{c}6.0 \\
(4.0-8.4)\end{array}$ & - & $\begin{array}{c}2.7 \\
(1.7-3.8)\end{array}$ & $\begin{array}{c}0.5 \\
(0-1.9)\end{array}$ & $\begin{array}{c}2.4 \\
(0.03-7.6)\end{array}$ & - & $\begin{array}{c}1.1 \\
(0.6-1.6)\end{array}$ & - & - & - & - & $\begin{array}{c}0.7(0.1- \\
1.6)\end{array}$ \\
\hline
\end{tabular}

PPM, permanent pacemaker; AF, atrial fibrillation; CVA, cerebrovascular accident; TBB, thromboembolism; TP, Tamponade; SVD, structural valve deterioration/degeneration; PVL, paravalvular leakage; NR, not reported. 